\title{
AN ASSESSMENT OF MATERNAL QUALITY OF LIFE IN THE POSTPARTUM PERIOD IN SOUTHERN BRAZIL: A COMPARISON OF TWO QUESTIONNAIRES
}

\author{
Carlos Zubaran, ${ }^{\text {K Katia Foresti, }}$ ' Marina Verdi Schumacher,II Mariana Rossi \\ Thorell,"II Lucia Cristina Muller,"II Aline Luz Amoretti'II
}

doi: 10.1590/S1807-59322009000800007

Zubaran C, Foresti K, Schumacher MV, Muller LC, Amoretti AL. An assessment of maternal quality of life in the postpartum period in southern Brazil: a comparison of two questionnaires. Clinics. 2009;64(8):751-6.

OBJECTIVES: To assess maternal quality of life (QoL) during the postpartum period and to compare the performance of two QoL questionnaires across a sample of 101 women in southern Brazil. To our knowledge, this is the first study that measures maternal quality of life during the postpartum period in Brazil.

INTRODUCTION: There is limited information about postpartum maternal quality of life in Brazil. The are no Portuguese versions of instruments specifically designed to measure quality of life during the postpartum period.

METHODS: Research participants completed the Portuguese version of the World Health Organization Quality of Life AssessmentBref (WHOQOL-BREF) and Multicultural Quality of Life Index (MQLI) questionnaires. The correlations between the MQLI and the discrete areas of WHOQOL-BREF were examined using Pearson Product-Moment Correlation Coefficients.

RESULTS: We report a significant correlation between the global MQLI and the four domains of the WHOQOL-BREF scores ( $p$ $<0.01)$. An analysis of variance revealed a significant difference in mean scores in the Psychological and Environment domains according to different socio-economic strata: $F(3,97)=3.81, p=0.012$ and $F(3,97)=4.03, p=0.01$, respectively.

DISCUSSION: The WHOQOL-BREF questionnaire may be more sensitive than the MQLI in detecting the impact of socioeconomic status on the QoL of postpartum women.

CONCLUSION: The sample of postpartum women evaluated in this study presented favorable QoL scores according to both the MQLI and WHOQOL-BREF questionnaires. Our results also indicate that the WHOQOL-BREF and the MQLI questionnaires have a significant correlation in terms of their assessments of postpartum mothers.

KEYWORDS: Quality of Life; Questionnaires; Postpartum Period; Postnatal Care; Brazil.

\section{INTRODUCTION}

The measurement of Quality of Life (QoL) has been evolving over the last 30 years and has now become a formal discipline with structured theoretical foundations and specific methodology. ${ }^{1}$ QoL has been increasingly recognized as an important outcome measure in both treatment studies and

${ }^{\text {I }}$ Western Sydney Area Health Service, Department of Psychiatry, Blacktown, Sydney, New South Wales Australia

II Universidade de Caxias do Sul - Caxias do Sul/RS, Brazil

III Hospital Santa Casa de Misericordia, Departamento de Pediatria - Porto Alegre/RS, Brazil

Email: zubaran_jr@yahoo.com

Tel: 13142860012

Received for publication on February 11, 2009

Accepted for publication on May 06, 2009 service evaluations. ${ }^{2}$ The assessment of QoL is widely used in clinical trials and in observational studies of health and disease. It is often used to evaluate interventions and the adverse effects of treatment as well as the impact of disease and other biological processes over time. ${ }^{3,4}$

However, there exists limited evidence regarding possible changes in QoL experienced by mothers during the postpartum period. During this time, women regularly report a series of physical symptoms such as fatigue, headache, dyspareunia, hemorrhoids and pain at multiple sites. ${ }^{5}$ Although these symptoms are frequently regarded as transient or inconsequential, they appear to be associated with functional maternal impairment and with poor emotional status. ${ }^{6}$ In fact, the risk of a major depressive disorder may also increase during the postpartum period. ${ }^{7}$ 
Until recently, no available tools were specifically designed to measure quality of life during the postpartum period. This situation was rectified by the development of two specific instruments. The Mother-Generated Index $(\mathrm{MGI})^{8}$ is a subjective tool, based on the Patient-Generated Index (PGI), which assesses the areas of a mother's life that may be affected during the month prior to delivery. The MGI was validated in Scotland ${ }^{8}$ and was subsequently evaluated through concurrent analysis using the Edinburgh Postnatal Depression Scale and the 12-item Short Form Health Survey (SF-12). ${ }^{9}$ Results from the initial MGI study demonstrated that mothers with MGI scores of 5 or lower had a significantly higher incidence of physical problems and depressive symptoms. ${ }^{10}$ The MGI facilitates the assessment of women's subjective status during late pregnancy $^{8}$ and postpartum. ${ }^{11}$

Another instrument specifically designed for use in the postpartum period is the Maternal Postpartum Quality of Life (MAPP-QOL) questionnaire, which is a self-administered instrument that measures quality of life during the early postnatal period..$^{12}$ The MAPP-QOL is composed of five domains and demonstrates convergent validity with measures of life satisfaction and mood states. ${ }^{12}$ A subsequent study used the MAPP-QOL to examine the postpartum quality of life of mothers of preterm, near-term, and full-term infants; the study revealed that the infant's gestational age at birth may influence maternal quality of life during the postpartum period. ${ }^{13}$

Since many tools are not available in Portuguese, the World Health Organization Quality of Life Assessment-Bref (WHOQOL-BREF) has been used in Brazil as a generic tool to assess QoL in women affected by conditions such as pelvic endometriosis, ${ }^{14}$ breast $^{15}$ and gynecological cancer, ${ }^{16}$ and infertility. ${ }^{17}$ In addition, the Multicultural Quality of Life Index (MQLI) is a generic instrument previously used in Brazil to assess QoL. ${ }^{18}$ The MQLI was developed as a brief, multidimensional and culturally sensitive scale. ${ }^{19}$

The present study aims to assess the quality of life of a sample of postpartum mothers in southern Brazil by evaluating and comparing the validity of two QoL assessment tools. Given the shortage of existing instruments designed for this purpose, the limitations of a generic approach using the WHOQOL-BREF and the MQLI are outweighed by the fact that, to the authors' knowledge, no previous study has measured maternal quality of life during the postpartum period in Brazil.

\section{MATERIALS AND METHODS}

\section{Sample Recruitment}

The study included a sample of 101 adult volunteers who gave birth in the General Hospital of the University of Caxias do Sul (UCS) in southern Brazil. This hospital is a higher education training facility and a regional center of excellence in obstetrics. Research volunteers were recruited via word of mouth by members of the research team while they were in the hospital in the days following delivery. Inclusion criteria were as follows: (a) patients were to be interviewed between their second and the twelfth weeks postpartum; (b) patients had to demonstrate the cognitive ability to understand and complete the questionnaires; (c) the mother must have delivered a living, healthy baby and (d) participants had to give their informed consent. Patients were excluded from the study either if they did not deliver in the General Hospital of the University of Caxias do Sul (UCS) or if they were already receiving treatment for depression.

Participants who fulfilled the above-mentioned criteria were interviewed once, in their own homes, between their second and twelfth weeks postpartum. All of the patients who agreed to participate in this investigation received assistance from the Brazilian public health system (SUS) and spoke Portuguese as their native language.

They were informed that they could voluntarily terminate their participation in the study at any time without any consequence to themselves or to the quality of their health care. All information obtained from research participants was kept confidential. All patients who were diagnosed with a depressive disorder according to the Structured Clinical Interview for DSM-IV Disorders (SCID) were promptly referred to the psychological outpatient clinic at the General Hospital of the University of Caxias do Sul for further assessment and treatment.

\section{Informed Consent}

This study was endorsed by the Institutional Ethics and Research Committee. All participants signed a consent form that declared their voluntary agreement with all the procedures involved in this project. Since a substantial number of patients were illiterate or semi-illiterate, all patients completed the Portuguese version of the PDSS with minimal guidance from trained examiners, who in turn followed standardized instructional procedures.

\section{Instruments}

Structured Clinical Interview for DSM-IV Disorders (SCID)

The SCID is a semi-structured interview that is widely used to assess major psychiatric diagnoses according to the Diagnostic and Statistical Manual of Mental Disorders (DSM) published by the American Psychiatric Association. ${ }^{20}$ Several studies have demonstrated the reliability and validity 
of the SCID..$^{21-23}$ The SCID has been translated into several languages, including Portuguese. An SCID assessment with a psychiatric patient may take 45 to 90 minutes, depending on the complexity of the patient's past psychiatric history and the subject's ability to clearly describe episodes related to current and past symptoms.

World Health Organization Quality of Life Assessment-Bref (WHOQOL-BREF)

The WHOQOL-BREF was developed by the World Health Organization as an abbreviated 26-item version of the WHOQOL-100 instrument. The WHOQOL-100 is used to measure quality of life across a variety of cultural settings. ${ }^{5}$ The WHOQOL-BREF is divided into four domains: Physical, Psychological, Social Relationships and Environment. Each domain score reflects an individual's perception of his or her quality of life in that particular area. Scores for each domain are scaled in a positive direction and the raw scores are converted to a metric on a scale from 0 to $100 .{ }^{24}$ Each domain requires that a minimum number of questions be answered in order to generate a score. Two items are examined separately: question 1 (Q1) asks about an individual's overall perception of quality of life and question 2 (Q2) asks about an individual's overall perception of his or her health. The WHOQOL-BREF has been validated across a wide range of languages, including Portuguese.?

\section{Multicultural Quality of Life Index (MQLI)}

The MQLI is a self-administered questionnaire that uses 10 items to assess global perception of quality of life in addition to physical and emotional well-being, self-care, occupational and interpersonal functioning, community and services support and personal and spiritual fulfillment. The Portuguese version of the MQLI has been tested in a mixed sample of patients and health professionals in Brazil. ${ }^{18}$ Spanish, ${ }^{25}$ Chinese, ${ }^{26}$ and Korean ${ }^{27}$ versions of the MQLI have also been validated. These studies have demonstrated that the MQLI is brief, easy to administer, internally consistent and substantially reliable. ${ }^{19}$

Research participants also completed a socio-economic status (SES) scale that had been developed and tested in Brazil. ${ }^{27}$ This instrument classified participants according to six socio-economic strata: Lower-Lower Class, Upper-Lower Class, Lower-Middle Class, Middle Class, Upper-Middle Class and Upper Class.

\section{Statistical Analysis}

Demographic analysis focused on each participant's age, educational background and the number of previous pregnancies and deliveries. To allow for comparative analyses, the variables had to be standardized across the different metrics and quantification systems. Metrics from the various scales were converted to $z$ scores and reversescaled so that all measures exhibited positive values as their highest possible scores. Multiple regression analyses were conducted to predict changes in the overall MQLI and WHOQOL-Bref scores. These analyses included SES, marital status and educational background as predictors. Relevant correlations among different assessment tools were examined using Pearson Product-Moment Correlation Coefficients. Statistical analysis was conducted with SPSS ${ }^{\circledR}$ software.

\section{RESULTS}

\section{Demographic Statistics}

The mean age of our participants was 25.6 years $(\mathrm{SD}=$ 7.04) with ages ranging from 14 to 42 years. Seventy-seven percent of the women were classified by interviewers as "White" according to Brazilian census criteria. Data on marital status revealed that $39 \%$ of the mothers were in de facto relationships (common-law marriages), $37 \%$ were married, $22 \%$ were single and 2\% were divorced. Most of the mothers $(66 \%)$ delivered vaginally, while $34 \%$ underwent a cesarean delivery. On average, research participants were interviewed 7.69 ( $\pm 1.7 \mathrm{SD})$ weeks after delivery.

Thirty-nine percent of the mothers in the study were giving birth to their first child; $32 \%$ were giving birth to their second or third child, and $30 \%$ were giving birth to subsequent children. Fourteen percent of the women had a previous history of miscarriage. Fifteen percent were smokers at the time of interview, and two percent of the participants reported regular alcohol consumption. Twentytwo percent had developed some kind of disease during pregnancy, and six percent presented complications during delivery. Seventeen percent of the mothers had a previous history of depression and fifty-seven percent had a family history of depression.

At the time of interview, 69 participants $(68.3 \%)$ were exclusively breastfeeding, 20 mothers $(19.8 \%)$ were partially breastfeeding and 12 mothers $(11.9 \%)$ were not breastfeeding. The socio-economic status (SES) of women in the sample was distributed as follows: $1 \%(n=1)$ were classified in the Lower-Lower Class, $13.9 \%(n=14)$ in the Upper-Lower Class, $71.3 \%(\mathrm{n}=72)$ in the Lower-Middle Class and $13.9 \%(n=14)$ in the Middle Class. No participants were categorized as being in the Upper-Middle and Upper Classes. Two participants (2\%) had a university degree; six participants $(5.9 \%)$ reported a partial university education; 21 participants $(20.8 \%)$ had completed high school; 26 
mothers $(25.7 \%)$ had a partial high school education; six mothers had completed primary school; and 40 mothers (39.6\%) had not completed primary school.

\section{Quality of Life Assessment}

The mean MQLI score was 76.37 ( $\mathrm{SD}=16.2)$. The WHOQOL-BREF mean scores and standard deviations are listed in Table 1. The Physical domain was the highest scoring WHOQOL-BREF area, while the Environment domain was the lowest scoring area. Results from Q1 revealed that mothers rated their overall quality of life as follows: very good (10\%), good (59\%), neither poor nor good $(27 \%)$, poor $(3 \%)$ and very poor $(2 \%)$. In Q2 participants rated their level of satisfaction with their health as follows: very satisfied (15\%), satisfied (52\%), neither satisfied nor dissatisfied (22\%), dissatisfied (10\%) and very dissatisfied (2\%).

Table 1 - WHOQOL-BREF mean and standard deviation (SD) per domain and coefficients of correlation with the overall MQLI score

\begin{tabular}{lccc}
\hline \multirow{2}{*}{ Domains } & \multirow{2}{*}{$\begin{array}{c}\text { WHOQOL-BREF } \\
\text { Mean (SD) }\end{array}$} & \multicolumn{2}{c}{ MQLI Correlations } \\
\cline { 3 - 4 } Physical (D1) & $71.07(15.94)$ & 0.75 & $<0.001$ \\
Psychological (D2) & $63.27(15.93)$ & 0.68 & $<0.001$ \\
Social (D3) & $67.19(19.2)$ & 0.56 & $<0.001$ \\
Environment (D4) & $60.59(12.16)$ & 0.57 & $<0.001$ \\
\hline
\end{tabular}

\section{Correlation Analyses}

The correlation $(r)$ between the overall MQLI score and the four domains of the WHOQOL-BREF are listed in Table 1. All correlations were significant and were associated with large effect sizes $(r \geq 0.5) .{ }^{28}$ In addition, a Spearman's rank correlation coefficient was computed between SES and educational status; this measurement revealed a significant correlation: $\rho=0.43, p<0.001$.

No correlation was identified between QoL and the length of the postpartum period according to the MQLI $(p=0.8)$ or as interpreted using three of the WHOQOL-Bref domains. A significant correlation with a small effect size was detected between the Environment domain and the length of the postpartum period ( $p=0.02, \mathrm{r}=0.23$ ).

\section{Analyses of Variance}

A one-way analysis of variance was conducted to evaluate the relationship between SES and QoL according to both the MQLI and the WHOQOL-BREF. The independent variable, SES, included 4 groups. The four domains of the WHOQOL-BREF were included as dependent variables. The results revealed a significant difference between the mean scores in the Psychological and Environment domains according to SES: $F(3,97)=3.81, p=0.012$ and $F(3$, $97)=4.03, p=0.01$, respectively. However, there was no significant difference in the mean scores of the MQLI among different SES groups: $F(3,97)=1.11, p=0.35$.

A one-way analysis of covariance (ANCOVA) was calculated to evaluate whether the mean scores of the MQLI and of each domain of the WHOQOL-Bref (dependent variables) may have varied across different SES levels (independent variable) after adjusting for educational background (covariate).

A preliminary analysis to evaluate the homogeneity-ofslopes assumption indicated that the relationship between the covariate and the dependent variables (WHOQOL-BREF domain scores) did not differ significantly as a function of the independent variable. The ANCOVA was significant only for the Psychological domain of the WHOQOL-Bref (F $(3,96)=3.5, p=0.02)$. The strength of the relationship (effect size) between SES and the Psychological domain was modest, as revealed by a partial $\eta^{2}$; when the education status of the research participants was held constant, SES accounted for $10 \%$ of the variance of the dependent variable.

A similar analysis was conducted using the MQLI scores as the dependent variable. Although a preliminary analysis evaluating the homogeneity-of-slopes assumption indicated that the relationship between the covariate (educational background) and the dependent variable did not differ significantly as a function of the independent variable, the ANCOVA was not significant either $(p=0.37)$.

\section{DISCUSSION}

The typical mother in this study is a white woman in her mid-twenties with a weak educational background and a lower SES; she is in a de facto relationship and is breastfeeding her first baby following a vaginal delivery. The majority of mothers in the study did not smoke or consume alcoholic beverages at the time of their deliveries. Some mothers had a previous history of depression, and most of them had a family history of depression.

The majority of participants assessed in this study reported satisfactory QoL according to the WHOQOLBREF. Similarly, most of the participants were "satisfied" with their health status according to this questionnaire. The results from the specific WHOQOL-BREF domains that are presented here show some congruence with crosssectional data obtained from a survey of adults carried out in 23 countries $(n=11,830)$. This survey reported QoL 
scores from a mixed Brazilian sample of healthy volunteers and patients assessed as part of the WHOQOL-BREF international trials. ${ }^{29}$

The mean QoL scores revealed by the MQLI in this study were slightly higher than the MQLI findings for a subsample of health care professionals $(n=30)$ that served as a control group during the initial trials of the MQLI in southern Brazil. ${ }^{18}$ Therefore, according to the MQLI, postpartum women in this area of southern Brazil report favorable QoL standards. The significant correlations $(p<0.01)$ between the MQLI and all of the WHOQOL-BREF domains, as well as the large size effect of these associations, further confirm the symmetry between the two QoL measurement tools.

Women recovering from delivery and experiencing the demands of a new baby, including breastfeeding, face a number of limitations in terms of their physical mobility, professional performance and social lives. The results of this study, however, indicate that many women sustain reasonable levels of QoL despite the challenges they may encounter during the postpartum period. A study using the MAPP-QOL to assess the QoL of 184 mothers revealed that the mean QoL scores increased from the first week postpartum to the third week. In addition, a significant increase was observed in terms of their health and functioning during the study. These findings suggest that the mothers in this sample were more satisfied with their lives in general as time passed. ${ }^{13}$ In a validation study of the Mother-Generated Index (MGI) in which 103 women were assessed during the postpartum period, mothers in the highest quartile group reported a sense of fulfilment and high self-esteem as well as good or improved relationships with family members. ${ }^{8}$

Since most of the women in the current study reported a low SES and limited level education, we assumed that the QoL standards in this sample would be limited by various socio-economic variables. A tendency towards lower QoL scores in lower socio-economic strata was also observed in a study in which 195 women were assessed using the MGI questionnaire. ${ }^{9}$ The subscale related to SES was also identified as a lower scoring area in the first week postpartum when mothers were assessed using the MAPPQOL. ${ }^{12}$ However, while the scores of two domains of the WHOQOL-BREF (Psychological and Environment) varied according to different SES, all other QoL measures failed to show any relationship between SES and QOL.

Since a significant correlation was observed between
SES and educational status in the present study, an ANCOVA was conducted to investigate whether the difference in mean QoL scores among different SES groups would persist after adjusting for educational background. The results of the ANCOVA conducted in this study demonstrated that, after educational background was held constant, a significant interaction between QoL and SES only persisted in the Psychological domain. Since similar outcomes were not obtained with scores from the MQLI and other WHOQOLBREF domains, both QoL questionnaires may, to some extent, be sensitive to the impact of educational background.

Factors such as older age and low levels of educational achievement have been correlated with low standards of QoL, ${ }^{30}$ while another study indicated that variables such as marital status, educational level and income did not influence WHOQOL-BREF scores in a sample of elderly volunteers recruited from public community health centers in Brazil. ${ }^{31}$ Although lower levels of education and income are associated with a significantly higher prevalence of risky health behaviors, ${ }^{32}$ research also demonstrates that income has a stronger mortality effect than education for both men and women. ${ }^{33}$ Further investigations into the interactions between SES and levels of educational attainment together with their distinct impacts on QoL standards should help improve the provision of healthcare to postpartum women in Brazil.

\section{CONCLUSION}

Results from the present study indicate that QoL measures are a valid and researchable element of community health care programs. The sample of postpartum women evaluated in this investigation presented favorable scores according to both the MQLI and WHOQOL-BREF questionnaires. This investigation also revealed that both the WHOQOL-BREF and the MQLI present significant score correlations in our assessment of mothers who experience the postpartum period. However, the WHOQOL-BREF questionnaire may be more sensitive to the impact of SES on the QoL of postpartum women. This strength may persist across specific domains despite different levels of educational attainment. Since there are few Portuguese questionnaires that are specifically designed to assess maternal QoL during the postpartum period, these findings represent a preliminary but pioneering evaluation of the $\mathrm{QoL}$ of postpartum mothers in southern Brazil. 


\section{REFERENCES}

1. Lohr K. Assessing health status and quality-of-life instruments: attributes and review criteria. Qual Life Res. 2002;1:193-205.

2. Wong JG, Cheung EP, Chen EY, Chan RC, Law CW, Lo MS, et al. An instrument to assess mental patients' capacity to appraise and report subjective quality of life. Qual Life Res. 2005;14:687-94.

3. Globe DR, Hays RD, Cunningham WE. Associations of clinical parameters with health-related quality of life in hospitalized persons with HIV disease. AIDS Care. 1999;11:71-86.

4. Schytt E, Lindmark G, Waldenstrom U. Physical symptoms after childbirth: prevalence and associations with self-rated health. BJOG. 2005;112:210-7.

5. Webb DA, Bloch JR, Coyne JC, Chung EK, Bennett IM, Culhane JF. Postpartum physical symptoms in new mothers: their relationship to functional limitations and emotional well-being. Birth. 2008;35:179-87.

6. Vesga-Lopez O, Blanco C, Keyes K, Olfson M, Grant BF, Hasin DS Psychiatric disorders in pregnant and postpartum women in the United States. Arch Gen Psychiatry. 2008;65:805-15.

7. Symon A, MacKay A, Ruta D. Postnatal quality of life: a pilot study using the Mother-Generated Index. J Adv Nurs. 2003;42:21-9.

8. Nagpal J, Sen Gupta Dhar R, Sinha S, Bhargava V, Sachdeva A, Bhartia A. An exploratory study to evaluate the utility of an adapted Mother Generated Index (MGI) in assessment of postpartum quality of life in India. Health Qual Life Outcomes. 2008;6:107.

9. Symon A, McGreavey J, Picken C. Postnatal quality of life assessment: validation of the Mother-Generated Index. BJOG. 2003;110:865-8.

10. Symon AG, Dobb BR. An exploratory study to assess the acceptability of an antenatal quality-of-life instrument (the Mother-generated Index). Midwifery. 2008; 24:442-50.

11. Hill PD, Aldag JC, Hekel B, Riner G, Bloomfield P. Maternal Postpartum Quality of Life Questionnaire. J Nurs Meas. 2006;14:205-20.

12. Hill PD, Aldag JC. Maternal perceived quality of life following childbirth. J Obstet Gynecol Neonatal Nurs. 2007;36:328-34.

13. Sepulcri Rde P, Amaral VF. Depressive symptoms, anxiety, and quality of life in women with pelvic endometriosis. Eur J Obstet Gynecol Reprod Biol. 2009;142: 53-6.

14. Rabin EG, Heldt E, Hirakata VN, Fleck MP. Quality of life predictors in breast cancer women. Eur J Oncol Nurs. 2008;12:53-7.

15. Vaz AF, Pinto-Neto AM, Conde DM, Costa-Paiva L, Morais SS, Esteves SB. Quality of life of women with gynecologic cancer: associated factors. Arch Gynecol Obstet. 2007;276:583-9.
16. Chachamovich J, Chachamovich E, Zachia S, Knauth D, Passos E. What variables predict generic and health-related quality of life in a sample of Brazilian women experiencing infertility. Hum Reprod. 2007;22:194652.

17. Zubaran CP, KN, Tarso D, Ioppi A, Mezzich J. Initial study for the development of the Portuguese version of the Multicultural Quality of Life Index. Arquivos Brasileiros de Psiquiatria, Neurologia e Medicina Legal 2004;98:1530-5.

18. Mezzich J, Cohen N, Ruiperez M, editors. A quality of Life Index: brief description and validation. International Congress of the International Federation for Psychiatric Epidemiology; 1996; Santiago de Compostela, Spain.

19. Steinberg M. Interviewers Guide to the Structured Clinical Interview for DSM-IV Dissociative Disorders (SCID-D). Washington, DC: American Psychiatric Press; 1994.

20. Gorman LL, O'Hara MW, Figueiredo B, Hayes S, Jacquemain F, Kammerer MH, et al. Adaptation of the structured clinical interview for DSM-IV disorders for assessing depression in women during pregnancy and post-partum across countries and cultures. Br J Psychiatry Suppl. 2004; $46: \mathrm{s} 17-23$

21. Del-Ben CM, Rodrigues CR, Zuardi AW. Reliability of the Portuguese version of the structured clinical interview for DSM-III-R (SCID) in a Brazilian sample of psychiatric outpatients. Braz J Med Biol Res. 1996;29:1675-82.

22. Riskind JH, Beck AT, Berchick RJ, Brown G, Steer RA. Reliability of DSM-III diagnoses for major depression and generalized anxiety disorder using the structured clinical interview for DSM-III. Arch Gen Psychiatry. 1987;44:817-20.

23. The WHOQOL Group. WHOQOL-BREF: Introduction, administration, scorin and generic version of the assessment. Geneva: World Health Organization; 1996.

24. Jatuff D, Zapata-Vega MI, Montenegro R, Mezzich JE. The multicultural quality of life index in Argentina: a validation study. Actas Esp Psiquiatr. 2007;35: 253-8.

25. Liu JS, Mezzich JE, Zapata-Vega MI, Ruiperez MA, Yoon G. Development and validation of the Chinese version of the Multicultural Quality of Life Index (MQLI-Ch). Cult Med Psychiatry. 2008;32:123-34.

26. Yoon G, Mezzich JE, Shin SK, Ruiperez MA, Zapata-Vega MI, Liu J. The Korean version of the Multicultural Quality of Life Index (MQLI$\mathrm{Kr}$ ): development and validation. J Immigr Minor Health. 2008;10:7380. 